\title{
Generalization of Extinguished Fear to Untreated Fear Stimuli after Exposure
}

\author{
Friederike Preusser', Jürgen Margraf' and Armin Zlomuzica*,I \\ 'Mental Health Research and Treatment Center, Ruhr-University Bochum, Bochum, Germany
}

\begin{abstract}
Exposure therapy is highly effective in treating excessive fear related to specific objects and/or situations. However, patients with anxiety disorders often display a generalization of fear responses toward conceptually and perceptually related stimuli and situations. It is unclear whether the beneficial effects of exposure on fear reduction toward treated fear stimuli can extend to untreated fear stimuli. Here, we investigated whether basic principles of extinction generalization apply to exposure. Spider-phobic participants were randomly assigned to either two sessions of exposure treatment $(n=23)$ with spiders or no-treatment $(n=24)$. Prior to and after treatment, behavioral approach tests (BATs) were conducted to examine avoidance, fear and disgust responses toward the treated phobic stimulus (spider as the extinction stimulus). Likewise, BATs with the untreated fear stimulus (cockroach) were conducted to dissect the generalization of treatment effects. Treatment was highly effective in increasing approach behavior toward both treated and untreated fear stimuli. Generalization of treatment effects were evident on the behavioral (approach distance during the BAT), subjective (fear levels during the BAT) and psychophysiological level (heart rate during the BAT). However, a stronger decline in disgust was only evident for the treated fear stimulus. Notably, the herein attained generalization effects were not context-dependent. Hence, exposure therapy for spider phobia was effective in reducing fear of untreated stimuli which share common fear-evoking characteristics with spiders but were never presented during the respective exposure treatment. These findings provide clinical evidence for extinction generalization across different fear-evoking stimuli mediated via exposure.

Neuropsychopharmacology (2017) 42, 2545-2552; doi:10.1038/npp.2017.1 19; published online 19 July 2017
\end{abstract}

\section{INTRODUCTION}

As supported by numerous meta-analytic studies (Butler et al, 2006; Hofmann and Smits, 2008; Norton and Price, 2007; Ruhmland and Margraf, 2001a,b,c), it is now widely established that exposure therapy represents the treatment of choice for anxiety disorders. The mechanisms underlying fear reduction and symptom relief during exposure therapy are not fully understood. Among different models, the fear extinction framework has evolved as the central candidate to explain exposure-induced fear reduction and symptom improvement (Vervliet et al, 2013). Fear extinction is not merely unlearning or erasure of the excitatory fear memory, but is best conceived of as the formation of a new inhibitory memory which competes with and ultimately inhibits the original fear memory (for a review, see Bouton, 2002). Hence, exposure might enable patients to acquire new inhibitory stimulus-safety associations which guide adaptive responses when dealing with the object of fear. In contrast to the excitatory fear memory, the formation of these new safety experiences during exposure is bound to contextual

\footnotetext{
*Correspondence: Dr A Zlomuzica, Mental Health Research and Treatment Center, Ruhr-University Bochum, Massenbergstreet 9-13, Bochum 44787, Germany, Tel: +49 23432 22347, Fax: +49 23432 |4369, E-mail: armin.zlomuzica@rub.de

Received 31 January 2017; revised 26 April 2017; accepted I June 2017; accepted article preview online 7 June 2017
}

details (Mineka et al, 1999; Mystkowski et al, 2002; Rodriguez et al, 1999), may these be interoceptive or exteroceptive (Bouton, 2002). This context-dependency of the inhibitory memory may ultimately hinder the generalization of therapy-induced effects to contexts or situations beyond the therapeutic setting.

Although the inhibitory learning model of extinction has become a valuable tool to evaluate novel routes to increase the efficacy of exposure-based treatments (Craske et al, 2008; Craske et al, 2014), it falls short of providing specific recommendations on how to deal with patients who express excessive fear responses to more than only one fear-inducing object. Thus far, it remains elusive whether exposure can induce fear reduction beyond the specific stimulus class dealt with during treatment. This, however, is crucial given that fear generalization constitutes a major hallmark feature of anxiety disorders (for a review, see Dymond et al, 2015).

Fear generalization refers to the process, whereby a stimulus acquires aversive properties due to both its' perceptual and/or conceptual similarity to another aversive stimulus. Since excessive fear generalization can account for the burden in life experienced by many anxiety patients, research on fear generalization has been on the rise in recent years (Dunsmoor and Paz, 2015; Dymond et al, 2015). Interestingly, studies related to its counterpart, namely extinction generalization, are scarce. Findings from 
Table I Demographic Characteristics as Well as Fear and Disgust Levels During the BAT at Pre-Assessment

\begin{tabular}{|c|c|c|c|c|c|}
\hline \multirow[t]{2}{*}{ Variable } & \multicolumn{2}{|c|}{$\begin{array}{l}\text { Treatment } \\
(n=23)\end{array}$} & \multicolumn{2}{|c|}{$\begin{array}{l}\text { No-Treatment } \\
\quad(n=24)\end{array}$} & \multirow[t]{2}{*}{$P$-value } \\
\hline & $\mathbf{M}$ & (SD) & $\mathbf{M}$ & (SD) & \\
\hline Age (years) & 23.17 & $(4.93)$ & 23.50 & $(3.19)$ & 0.788 \\
\hline Gender (female) & $87 \%$ & & $95.8 \%$ & & 0.276 \\
\hline Disgust sensitivity (FEE) & 86.53 & $(18.66)$ & 88.63 & $(16.42)$ & 0.684 \\
\hline DASS (total) & 15.04 & $(13.37)$ & 10.29 & $(5.35)$ & 0.114 \\
\hline Depression & 3.70 & $(5.60)$ & 2.08 & $(2.15)$ & 0.196 \\
\hline Anxiety & 3.57 & $(3.80)$ & 1.92 & $(1.67)$ & 0.059 \\
\hline Stress & 7.78 & $(5.23)$ & 6.29 & $(3.14)$ & 0.240 \\
\hline \multicolumn{6}{|l|}{ BAT spider (pre) } \\
\hline Fear & 68.86 & $(19.88)$ & 70.00 & $(20.38)$ & 0.848 \\
\hline Disgust & 69.77 & $(21.98)$ & 84.17 & $(15.08)$ & $0.012^{\mathrm{a}}$ \\
\hline \multicolumn{6}{|l|}{ BAT cockroach (pre) } \\
\hline Fear & 51.82 & $(27.24)$ & 53.48 & $(24.38)$ & 0.827 \\
\hline Disgust & 62.73 & $(27.17)$ & 71.09 & $(21.42)$ & 0.246 \\
\hline
\end{tabular}

conditioning studies indicate that fear reduction following a fear extinction procedure does in fact generalize from the extinction stimulus to other perceptually (Vervliet et al, 2005; Vervliet et al, 2004) and conceptually related stimuli (Vervoort et al, 2014), with this extinction generalization effect being only evident when the original fear stimulus is subjected to extinction.

Only a few treatment studies investigated extinction generalization in the therapy context (Byrne et al, 2015; Pace-Schott et al, 2012; Rowe and Craske, 1998a,b), but focused on therapy-induced fear reduction across different exemplars of the treated stimulus (eg, same stimulus class). Despite its clinical importance, no study has yet investigated the basic principles of extinction generalization toward untreated fear stimuli in the exposure context (eg, different stimulus class).

The present study sought to fill these gaps. Here, participants with spider phobia were randomly assigned to either treatment for spider fear or no-treatment. Since fear of spiders has been shown to covary with fear of cockroaches (Davey, 1991) and both stimuli tend to elicit a comparable repertoire of emotional, cognitive and behavioral responding, cockroaches were employed as the test stimulus to measure extinction generalization. Given that extinction is context-dependent and the contextual details during extinction can serve to retrieve stimulus-safety associations (Bouton, 2002), we further employed an exteroceptive context change (ie, rooms which differed in various details) to examine whether the clinical analogue of extinction generalization is modulated by changes in contextual features. Finally, in light of a growing body of research which indicates that maladaptive responses in anxiety disorders can be generated and maintained not only by fear but also disgust (Olatunji and McKay, 2007a; Woody and Tolin, 2002), we focused on therapy-induced changes in both emotions as complementary measures since this is of particular relevance in spider phobia (de Jong and Muris, 2002).

\section{MATERIALS AND METHODS}

\section{Participants}

Seventy seven individuals were recruited by announcements in social media networks or bulletin board notices at the campus of the Ruhr-University to participate in a study to treat their spider fear. Participants with any neurological or neuropsychiatric conditions were not eligible for participation. Thirty participants were excluded from data analysis because they displayed insufficient fear of cockroaches (ie, indicated by a score of 10 on the Behavioral Approach Test at preassessment). Our analytic sample thus comprised $N=47$ participants who were either assigned to the treatment (ie, received two sessions of exposure treatment for spider fear; $n=23$ ) or no-treatment condition (ie, received no exposure or other treatment; $n=24$ ). Demographic characteristics are displayed in Table 1 . The study was approved by the local ethics' committee of the Ruhr-University Bochum and carried out in accordance with the Declaration of Helsinki. All participants provided written informed consent and were reimbursed with 4 course credits or 40 euros.

\section{Measures}

Diagnostic interview. Diagnosis of spider phobia was ascertained with the short diagnostic interview for mental disorders (Mini-DIPS; Margraf, 1994), which is based on DSM-IV criteria.

Questionnaires. Selected items from the Depression, Anxiety, Stress Scales (DASS; Lovibond and Lovibond, 1995) were used to measure acute symptoms of Depression, Anxiety and Stress on a 4-point Likert scale $(0=$ did not apply to me at all; $3=$ applied to me very much). Individual differences in disgust sensitivity were assessed with the German questionnaire for the assessment of disgust sensitivity ('Fragebogen zur Erfassung der Ekelempfindlichkeit' (FEE; Schienle et al, 2002)), which comprises 37 items scored on a 5-point Likert scale ( $1=$ not disgusting, $5=$ very disgusting). Spider-related fear and beliefs were measured using three questionnaires: (1) The German version of the Fear of Spiders Questionnaire (FSQ; Rinck et al, 2002), which consists of 18 items and is scored on a 7-point Likert scale; (2) the 31-item Spider Phobia Questionnaire (SPQ; Hamm, 2006) in its' dichotomous format, with items being judged as either true or false; and (3) the Spider Beliefs Questionnaires (SBQ; Pössel and Hautzinger, 2003), which comprises 48 items measuring dysfunctional beliefs on visual analogue scales from 0 ( = not at all) to 100 (= completely). On each of these questionnaires, higher scores correspond to greater spider fear and/or unrealistic beliefs. 
Behavioral approach test. The behavioral approach test (BAT) was employed to measure avoidance and fear of a spider (Tegenaria domestica, $1 \mathrm{~cm}$ ) and a cockroach (Blaptica dubia, $4 \mathrm{~cm}$ ) by having participants approach the fear stimulus, which was placed in a plastic container at the far end of the room, as fast and close as possible, ie, until fear becomes unsustainable. The BATs were scored behaviorally in terms of approach distance $(0 \quad(=$ refused to enter the room) to 10 (=touched the spider with the fingertip)) as well as subjectively in terms of fear and disgust levels (SUDs) at the closest proximity to the fear stimulus tolerable.

Fear and disgust levels. The Subjective Units of Distress Scale (SUDS; Wolpe, 1973) with scores from 0 (= no fear) to 100 ( $=$ excessive fear) served as the primary measure of fear and disgust during the exposure treatment and the BATs.

Psychophysiology. During the BATs and the exposure sessions, heart rate (HR), averaged across $5 \mathrm{~s}$ intervals, was measured non-invasively with a Polar electrode belt, which was fitted around participants' chest, and a wrist watch receiver unit (Polar RS800 CX), which stored the data.

\section{Exposure Treatment}

Treatment consisted of two $1 \mathrm{~h}$ sessions according to a slightly modified version of the protocol by Öst (1997). Exposure comprised a hierarchy of 7 steps, which involved (1) watching the spider in a glass, (2) watching it crawl in a plastic container, (3) touching it with a pencil, (4) a latex glove and (5) fingertip, and finally (6) letting it walk on the hand and (7) arm. These steps were first accomplished with a vibrating spider (Pholcidae, $1 \mathrm{~cm}$ ), and then repeated with the house spider (ie, the same one used for the BATs). The experimenter first modeled each step to the participant, after which the participant performed the step himself. Fear levels (SUDs) were collected at the beginning of each step and collected continuously. Each step was repeated until a SUDs of 30 or below was obtained. Exposures were terminated when $60 \mathrm{~min}$ had passed or when all steps had been completed with both spiders.

\section{Experimental Design and Procedure}

Participation in the study comprised three appointments: a screening session (session 1), pre-assessment (session 2), and post-assessment (session 3). An outline of the study design is displayed in Figure 1.

Screening session. This session was conducted one week prior to pre-assessment. Participants completed the diagnostic interview for spider phobia, the demographic questionnaire, the DASS, the Disgust sensitivity and the spider fear-related questionnaires (ie, FSQ, SPQ, SBQ).

Pre-assessment. Upon arrival, participants were fitted with the HR equipment and a 5-min recording of resting HR was undertaken. Subsequently, participants received instructions on the BAT and the use of the SUDs scale. Participants were then escorted to the respective rooms, in which the two BATs were conducted. After the BATs, the treatment group accomplished the first exposure session.

Post-assessment. On an average interval of 4 days $(\mathrm{SD}=$ 2.8), the post-assessment was conducted. After a 5-min resting HR recording and rehearsal of the BAT instructions, the no-treatment group accomplished the two BATs. By contrast, the experimental group first underwent another exposure session, after which another 5-min resting HR was recorded, the BAT instructions rehearsed, and the BATs conducted. During the post-assessment BATs, participants

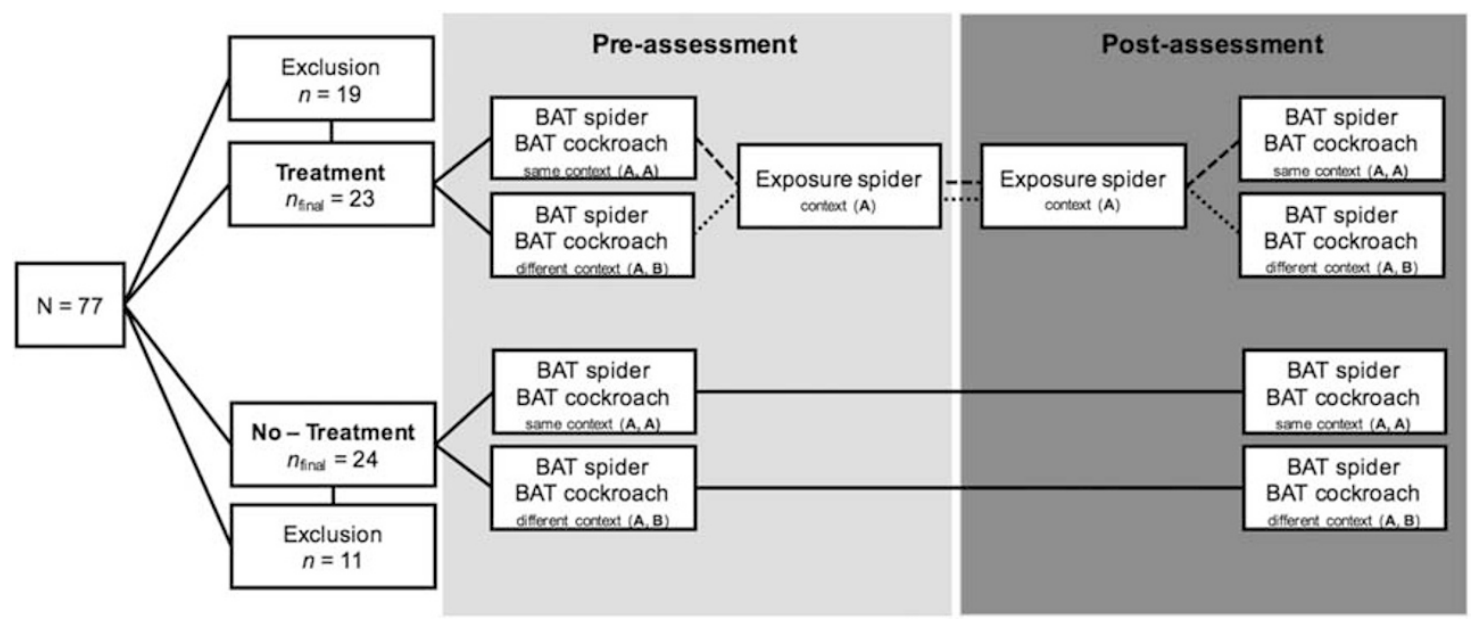

Figure I Brief outline of the experimental design. Participants were assigned to a treatment or a no-treatment group. Only the treatment group engaged in two exposure sessions with spiders in context A. Therapy-induced changes in fear, disgust and avoidance of a house spider were assessed with behavioral approach tests (BATs) prior to (pre-assessment) and after treatment (post-assessment). Additional BATs with cockroaches (Blaptica dubia, $4 \mathrm{~cm})$ as the untreated stimulus were conducted to assess extinction generalization. The order of BATs with spiders and cockroaches was counterbalanced across participants. The BAT with spiders was always conducted in the treatment room (context A), while the BAT with cockroaches was either conducted in the treatment room (ie, context A; treatment group: $n=14$; no-treatment group: $n=12$ ) or an adjacent, novel room (ie, context B; treatment group: $n=9$; notreatment group $n=12$ ). Contexts $A$ and $B$ differed in size and shape, illumination, decoration, and furniture, as well as in size and color of the plastic containers used for BATs (adapted from Mineka et al, 1999; Mystkowski et al, 2002; Rodriguez et al, 1999). 
indicated their fear and disgust at the same distance to the respective fear stimulus they had accomplished at the preassessment (=defined as the initial approach distance). Thereafter, participants continued with the BATs if their fear was not yet unsustainable and indicated their level of fear and disgust at the closest proximity tolerable (=defined as the final approach distance). At the end of session 2, participants completed the spider fear-related questionnaires (FSQ, SPQ, SBQ) and were fully debriefed.

\section{Statistical Analyses}

Data were analyzed using SPSS Version 23 (Armonk, NY, USA). The BATs in terms of approach distance (behavioral level), fear and disgust at the final and initial approach distances (subjective level), as well as heart rate (psychophysiological level) were analyzed separately for treatment effectiveness (spider) and extinction generalization (cockroach). Mixed ANOVAs or MANOVAs were conducted as appropriate: Group (treatment vs no-treatment) and context (where appropriate; same $v s$ different) were used as betweensubject factors and time (pre-assessment $v s$ post-assessment) as within-subjects factor. In all heart rate analyses, heart rate averaged across the respective BAT trial served as the dependent measure. Resting heart rate prior to the first BAT as well as its square were entered as covariates in mixed ANCOVAs. Here, due to technical errors, data from only 37 participants (treatment group: $n=15$; no-treatment group: $n=22$ ) was available. A result was considered significant at an alpha level of $P<0.05$.

\section{RESULTS}

\section{Pre-Exposure}

The treatment and no-treatment group were no different in terms of the proportion of participant exclusions due to insufficient fear of cockroaches $(P=0.235)$. As depicted in Table 1, the treatment group did not differ from the no-treatment group in any demographic variables such as age, gender, and their scores on any subscale of the DASS. Importantly, groups were comparable with regard to their subjective fear and disgust levels on the BATs with spiders and cockroaches (cf. Table 1) and their scores on the spider fear-related questionnaires (cf. Table 2) at pre-assessment.

\section{Treatment Effectiveness (Spider)}

Spider fear-related questionnaires. The MANOVA on the FSQ, SPQ and SBQ revealed significant main effects for time, $F(3,43)=36.049, P<0.001$, and group, $F(3,43)=9.878$, $P<0.001$ as well as their interaction, $F(3,43)=35.978$, $P<0.001$. As shown in Table 2, only the treatment group exhibited a decline in their scores over time $(P<0.001)$, which were significantly lower than those of the no-treatment group at post-assessment $(P<0.001)$.

Behavioral scores on the BATs. There were significant improvements on the BAT from the first to the second assessment (main effect for time, $F(1,45)=104.51 P<0.001$ ). As expected, the amount of improvement over time was subjected to group differences [interaction, $F(1,45)=77.263$,
Table 2 Pre- and Post-Assessment Scores on the Spider Fear-Related Questionnaires

\begin{tabular}{|c|c|c|c|c|c|}
\hline \multirow[t]{2}{*}{ Variable } & \multicolumn{2}{|c|}{ Treatment $(n=23)$} & \multicolumn{2}{|c|}{ No-Treatment $(n=24)$} & \multirow[t]{2}{*}{$P$-value } \\
\hline & $\mathbf{M}$ & (SD) & $M$ & (SD) & \\
\hline \multicolumn{6}{|l|}{ FSQ } \\
\hline Pre & 69.35 & $(23.74)$ & 74.42 & $(15.95)$ & 0.393 \\
\hline Post & 24.91 & $(16.4 I)$ & 72.09 & $(16.92)$ & $<0.001^{\mathrm{a}}$ \\
\hline \multicolumn{6}{|l|}{$S P Q$} \\
\hline Pre & 20.00 & $(4.53)$ & 20.08 & $(4.69)$ & 0.951 \\
\hline Post & 9.87 & $(4.94)$ & 20.26 & $(4.87)$ & $<0.001^{a}$ \\
\hline \multicolumn{6}{|l|}{$S B Q$} \\
\hline Pre & 58.76 & $(19.21)$ & 61.06 & $(16.28)$ & 0.659 \\
\hline Post & 18.63 & $(14.24)$ & 59.32 & $(19.57)$ & $<0.001^{a}$ \\
\hline
\end{tabular}

Abbreviations: FSQ, Fear of Spiders Questionnaire; SPQ, Spider Phobia Questionnaire; SBQ, Spider Beliefs Questionnaire.

a Significant between-group differences, with $P<0.00$ I.

$P<0.001)$ ], with the treatment group showing a greater increase in approach behavior toward spiders than the notreatment group. Results are displayed in Figure 2 (left).

Fear and disgust levels during the BATs. Fear at either the initial or final approach distance were analyzed separately, using mixed ANOVAs with time as within-subjects factor and group as between-subjects factor. Due to missing data of $n=3$ participants in the no-treatment group, analysis of fear and disgust levels at the initial approach distance was restricted to only $N=44$ participants. Data were missing because these participants were not able to re-attain their pre-assessment BAT approach distance at the postassessment BAT (ie, showed a closer proximity to the spider at pre-compared to post-assessment), with a return to the pre-BAT distance being a prerequisite for assessing fear/ disgust at this approach distance.

Both analyses yielded significant main effects for time (initial: $F(1,42)=109.67, P<0.001$; final: $F(1,45)=47.627$, $P<0.001$ ), group (initial: $F(1,42)=28.259, P<0.001$ ); final: $F(1,45)=19.917, P<0.001)$, as well as their interaction (initial: $F(1,42)=91.924, P<0.001)$; final: $F(1,45)=42.007$, $P<0.001)$. As shown in Figure $3 a$, the treatment group showed a stronger decline in fear as compared to the no-treatment group.

Likewise, both mixed ANOVAs on disgust levels at the initial and final approach distance indicated significant main effects for time (initial: $F(1,42)=51.645, P<0.001$; final: $F(1,45)=38.932, P<0.001$ ), group (initial: $F(1,42)=39.947$, $P<0.001$; final: $F(1,45)=39.072, P<0.001)$ and their interaction (initial: $F(1,42)=33.519, \quad P<0.001$; final: $F(1,45)=29.172, P<0.001)$. As shown in Figure $3 c$, disgust was subjected to a stronger decline in the treatment group compared to the no-treatment group.

Heart rate during the BATs. Heart rate (adjusted for the covariates in the model) during the BAT at pre- and 

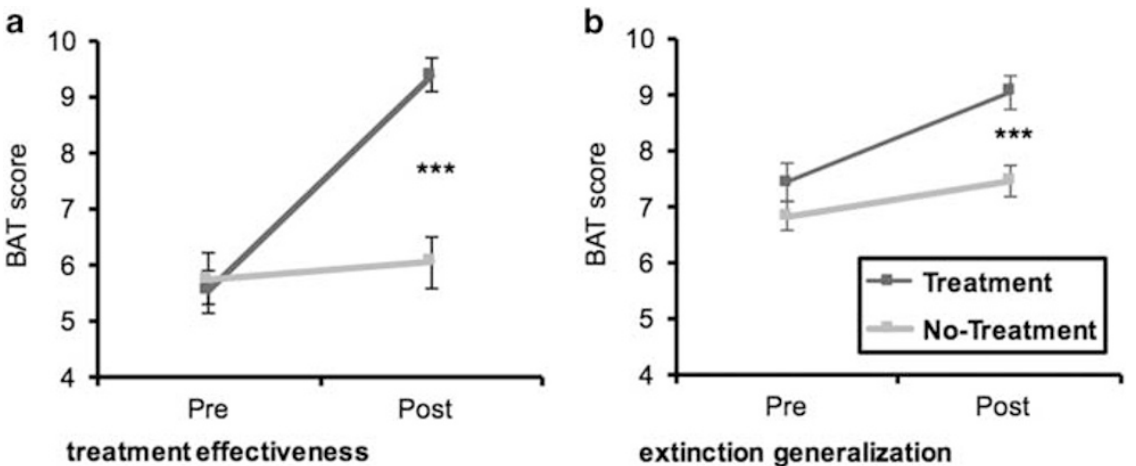

Figure 2 Results of the BAT with spiders (treatment effectiveness: left; a) and cockroaches (extinction generalization: right; b) in terms of approach distance. Scores are displayed as Means \pm I SEM. $* * * * P<0.001$.

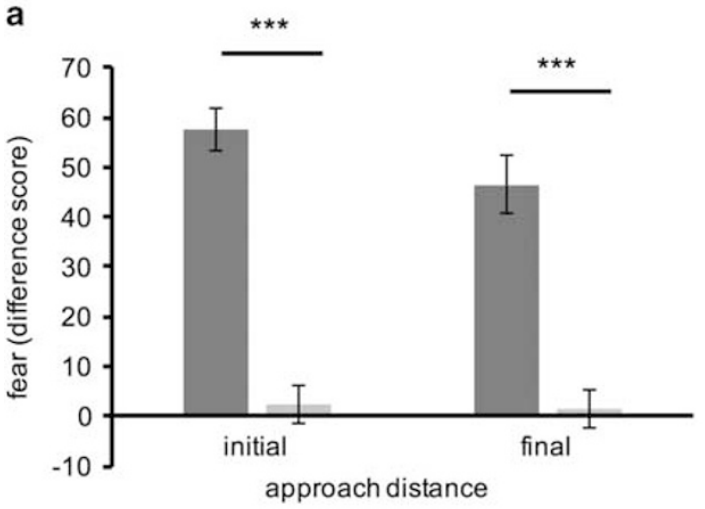

treatment effectiveness (fear)

C

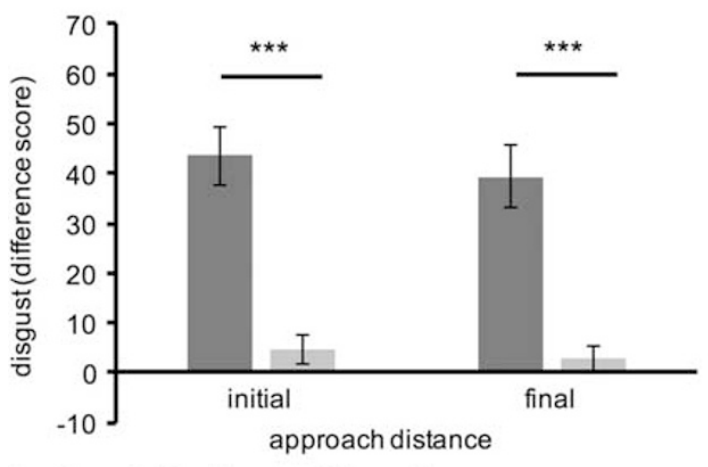

treatment effectiveness (disgust) b

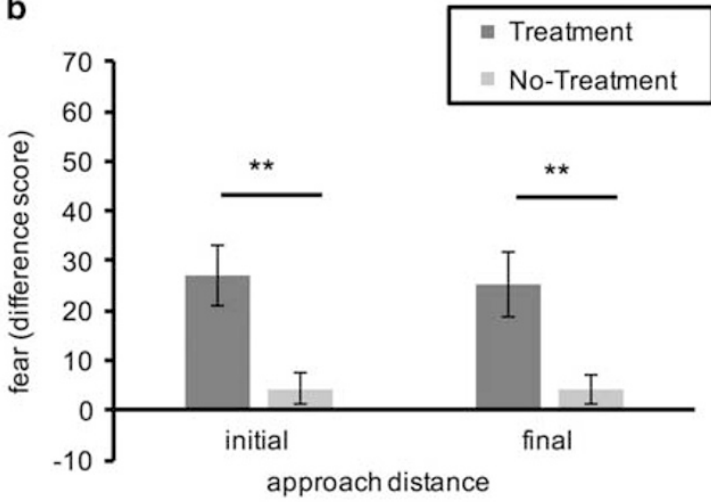

extinction generalization (fear)

d

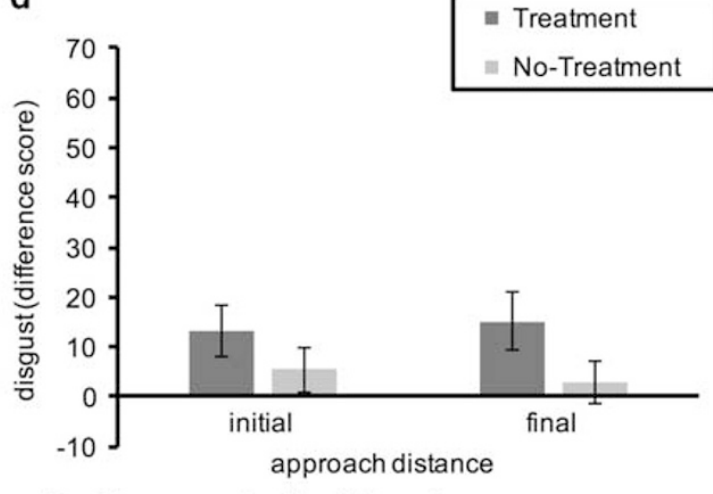

extinction generalization (disgust)

Figure 3 Represents the reduction in fear (top; $a$ and b) and disgust (bottom; $c$ and d) from pre- to post-assessment (difference score) at the initial and final approach distance, separately for treatment effectiveness (left; $a$ and $c$ ) and extinction generalization (b and d). Greater scores correspond to greater reduction from pre-to post-assessment. Scores are displayed as Means \pm I SEM. $* * * * P<0.00$ I, $* * * P<0.0$ I.

post-assessment were $M=95.49(\mathrm{SE}=2.64)$ and $M=82.90$ $(\mathrm{SE}=2.57)$ for the treatment and $M=98.98(\mathrm{SE}=2.17)$ and $M=93.93$ ( $\mathrm{SE}=2.11$ ) for the no-treatment group. Again, the significant interaction between group and time, $F(1,33)$ $=5.309, P=0.028$, revealed that heart rate decreased more readily from the first to the second BAT in the treatment group compared to the no-treatment group.

\section{Extinction Generalization (Cockroach)}

Behavioral scores on the BAT. Similar to the BAT with spiders, the main effects for time and group attained significance, both $F(1,43) \geqslant 8.819, P \leqslant 0.005$. As shown in Figure 2 (right), the group $\times$ time interaction, $F(1,43)=$ $7.846, P=0.008$, revealed that the treatment group displayed a greater increase in approach behavior toward cockroaches from the first to the second assessment than the notreatment group. Importantly, there was no main effect for or any interaction with context (all $P>0.18$ ).

Fear and disgust levels during the BAT. Fear levels at either the initial or final approach distance were analyzed separately with mixed ANOVAs with time as within-subjects factor and group as well as context as between-subjects 
factors. Fear declined significantly over time at the initial, $F(1,43)=20.993, P<0.001$, and final approach distance, $F(1,43)=19.516, P<0.001$. In both analyses, the decline in fear over time was subjected to group differences (interaction: initial $F(1,43)=11.089, P=0.002$; final: $F(1,43)=$ 10.204, $P=0.003$ ), with the treatment group displaying a stronger decline in fear as compared to the no-treatment group (cf. Figure 3b). In none of these analyses did any effects for context attain statistical significance $(P>0.15)$.

Likewise, significant main effects for time emerged in the mixed ANOVAs on disgust levels at the initial, $F(1,43)$ $=8.118, P=0.007$, and final approach distance, $F(1,43)$ $=6.364, P=0.015$. However, as shown in Figure $3 \mathrm{~d}$, the treatment group did not show a stronger decline in disgust over time, neither at the initial, $P=0.22$, nor at the final approach distance, $P=0.09$. Again, in none of these analyses were effects for context evident (all $P>0.08$ ).

Heart rate during the BATs. Adjusted mean heart rate was $M=95.66(\mathrm{SE}=2.27)$ and $M=83.18(\mathrm{SE}=2.60)$ for the treatment group as well as $M=94.73 \quad(\mathrm{SE}=1.83)$ and $M=89.97 \quad(\mathrm{SE}=2.1)$ for the no-treatment group during the BATs at pre- and post-assessment, respectively. The significant group $\times$ time interaction, $F(1,31)=7.226$, $P=0.011$, indicated a stronger decline in heart rate over time for the treatment group. Again, no effects for context were evident (all $P>0.47$ ).

\section{DISCUSSION}

Participants who underwent exposure treatment for spider fear showed significant reductions in fear of spiders from pre- to post-assessment. Most importantly, substantial reductions in fear of and increased approach behavior toward untreated fear stimuli (ie, cockroaches) were also observed although its fearful characteristics had not been formally extinguished during exposure. These beneficial effects of exposure on generalization of treatment effects were coherently evident on the subjective (fear levels during the BAT), behavioral (approach distance) as well as on the psychophysiological (heart rate during the BAT) level. By contrast, the no-treatment group did not demonstrate the same amount of improvement, neither with spiders nor with cockroaches.

Our results extend existing translational findings on extinction generalization by showing that exposure reduces not only fear toward other exemplars of the treated phobic stimulus (Byrne et al, 2015; Pace-Schott et al, 2012; Rowe and Craske, 1998a,b) but also toward untreated fear stimuli (ie, cockroaches). Our findings are in accordance with basic and clinical research on fear generalization in humans (Dunsmoor and Murphy, 2015; Dunsmoor and Paz, 2015; Dymond et al, 2015). From the conditioning perspective, exposure is considered as a form of corrective learning during which patients learn to respond adaptively to initially fear-evoking stimuli (CS). Successful exposure is thus accompanied by the acquisition of new adaptive responses associated not only with the treated CS but also with the untreated CS, which resembles the treated CS along various dimensions. The presentation of untreated stimuli, which share similar features with the treated stimuli, seems to be

sufficient to elicit similar adaptive responses, which have been recently associated with the treated stimuli. The extent to which untreated stimuli evoke similar adaptive responses after exposure is dependent on the relative similarity between treated and untreated stimuli (Dunsmoor and Murphy, 2015; Dunsmoor and Paz, 2015; Dymond et al, 2015). Our study provides novel insight to current research on fear generalization in various central aspects.

In contrast to existing exposure therapy studies, we used test stimuli to measure extinction generalization, which are not exclusively perceptually related (such as other exemplars of the phobic object, which differ in size and texture; see (Byrne et al, 2015; Pace-Schott et al, 2012; Rowe and Craske, 1998a,b)) to the phobic stimulus. Fear of spiders has been shown to be correlated with fear of cockroaches in spiderfearful participants and both stimuli are capable of evoking disgust (Davey, 1991; Matchett and Davey, 1991). The presumed similarity between these stimuli is based on their 'fear-evoking characteristics' (Davey, 1991). These 'fearevoking characteristics' might be related to various perceptual features of spiders and cockroaches such as legginess, sudden movements, or speediness (Davey, 1991). Likewise, similarities in common cognitive misconceptions, including their harm-related character, uncontrollability or unpredictability, have been isolated for spiders (Arntz et al, 1993), but might also apply to cockroaches. Previous research on this topic indicates that spider-fearful individuals tend to differ in their reports on which of these characteristics are most frightening (Arntz et al, 1993; Davey, 1991). Therefore, the determination of a common basis on the fear-evoking characteristics of spiders and cockroaches might be difficult to achieve. Future exposure studies might provide more substantial insights into perceptual versus conceptual or category-based generalization by applying approaches from conditioning studies (eg, Dunsmoor and Murphy, 2015), which used pre-existing animal categories or manipulated relatedness of the cues involved by typicality or frequency of occurrence (Dunsmoor and Murphy, 2014). Other approaches to assess generalization in exposure therapy could make use of individual ratings of the relatedness of various insect stimuli and use those stimuli which are closer/further away from the phobic stimulus. Furthermore, fear generalization based on differences/similarities in intensity dimension, category-based or symbolic fear generalization could be addressed in future clinical studies (Dymond et al, 2015). However, the translation of findings from the conditioning literature to exposure is far from self-evident and might suffer from methodological shortcomings. As indicated, there are profound individual differences in self-report on the relatedness between spiders and other insects or animals (Arntz et al, 1993; Davey, 1991). Furthermore, generalization in the exposure context in specific phobia might sometimes be difficult to incorporate because some stimuli cannot easily be applied to standardized experimental procedures. For instance, the advantage of using cockroaches (instead of snakes and rats) as test stimuli is not only justified because they induce similar fear levels as spiders but also because these stimuli can easily be applied to exposure and experimental protocols already exist (Botella et al, 2010). These shortcomings apply to animal-related phobia and other specific phobia. 
In contrast to previous research on the generalization of exposure effects, we assessed fear and disgust as complementary measures. Although exposure was highly effective in decreasing fear responses toward both spiders and cockroaches, the decline in disgust did not generalize equally to cockroaches. This effect might be due to the underlying learning processes and mechanisms which contribute to the acquisition and extinction of fear and disgust (Hofmann et al, 2010). The acquisition and maintenance of fear and disgust is based on two different forms of associative learning. Disgust is related to evaluative conditioning, while fear is more reliant on expectancy learning with respect to the association of CS and UCS (Woody and Teachman, 2000). Findings from conditioning studies indicate that responses based on evaluative conditioning (such as disgust or dislike) are more resistant to extinction (Baeyens et al, 2005). This might explain why fear shows a stronger decline as compared to disgust during exposure (Olatunji et al, 2007b; Smits et al, 2002) and why heightened disgust responses after successful exposure may persist in some patients despite substantial fear reduction. This underlines the importance of considering disgust as another important dimension to be modified. Here interventions such as counterconditioning and US revaluation have been proposed as promising alternatives to alter existing disgust responses during exposure (see Ludvik et al, 2015).

Our study also extends previous research on extinction generalization by shedding light on the role of the context. Regardless of whether the BAT was conducted in the familiar (ie, treatment) or the novel context, the treatment group demonstrated substantial fear reduction and increased approach behavior toward the untreated fear stimulus. Thus, in contrast to the context-dependency of extinction (Bouton, 2002), the generalization of exposure effects was not contextspecific. The interpretation of these findings should nonetheless be undertaken with caution due to the limited sample size in context analyses and other shortcomings. In particular, the therapist himself might become a safety signal during exposure (Barlow, 1988) and thus construes a strong contextual element, which was not manipulated. Likewise, the BAT procedure in itself might represent a salient feature prompting generalization across contexts.

It needs to be considered that apart from whether or not groups had received exposure, other factors (ie, more social interaction with the experimenter in the treatment group) might have led to the group differences observed. However, since we used a standardized exposure protocol (adapted from Öst, 1997) and refrained from additional therapeutic interventions (ie, cognitive instructions or tools to encourage patients) in the treatment group, we are confident that these factors are unlikely to explain the stronger generalization effects in this group.

Our approach represents a possible novel translational tool to examine generalization in the therapy context and might provide new input to current basic and clinical research on generalization of fear and its extinction. Specifically, conditioning studies have shown that the generalization of extinction is critically dependent upon the stimulus subjected to extinction (Vervliet et al, 2004; Vervoort et al, 2014): whereas extinguishing a generalized stimulus does not lead to fear reduction toward the original fear stimulus, extinguishing the original fear stimulus does concomitantly reduce fear of the generalized stimuli. Therefore, the question as to whether any generalization of treatment effects is still attained if participants were subjected to treatment with the less fearinducing stimulus remains to be addressed. Future studies using our approach might hence unravel whether exposure necessarily needs to be conducted with a specific object of fear. Thus, one could compare the effects of exposure with spiders $v s$ cockroaches and examine fear (and disgust) to the other stimulus class. Furthermore, one could employ our approach to identify pharmacological and behavioral strategies as add-ons to optimize generalization of exposure therapy effects across different fear-evoking stimuli.

In conclusion, our findings provide first evidence for generalization of exposure effects in spider phobia to other similar fear-evoking stimuli, ie, cockroaches. While it remains to be explored whether extinction generalization toward 'fearevoking characteristics' of spiders and cockroaches (Davey, 1991) is based on perceptual or non-perceptual similarities, our findings nonetheless indicate that exposure with one phobic object also leads to fear reductions when being confronted with other phobic-related fears. This suggests a certain degree of transfer of coping capabilities acquired during exposure toward similar untreated fear stimuli.

\section{FUNDING AND DISCLOSURE}

This study was supported by the Deutsche Forschungsgemeinschaft (DFG) with grant ZL 59 2/2 awarded to Armin Zlomuzica, Jürgen Margraf and Silvia Schneider. The authors declare no conflict of interest.

\section{AUTHOR CONTRIBUTIONS}

FP, JM, AZ designed the research. FP performed the research. FP and $\mathrm{AZ}$ analyzed the data. FP and $\mathrm{AZ}$ wrote the manuscript. All authors approved the final manuscript.

\section{REFERENCES}

Arntz A, Lavy E, van den Berg G, van Rijsoort S (1993). Negative beliefs of spider phobics: a psychometric evaluation of the spider phobia beliefs questionnaire. Adv Behav Res Ther 15: 257-277.

Baeyens F, Diaz E, Ruiz G (2005). Resistance to extinction of human evaluative conditioning using a between-subjects design. Cogn Emot 19: 245-268.

Barlow DH (1988). Anxiety and its Disorders: The Nature and Treatment of Anxiety and Panic. Guilford Press: New York.

Botella C, Breton-Lopez J, Quero S, Banos R, Garcia-Palacios A (2010). Treating cockroach phobia with augmented reality. Behav Ther 41: 401-413.

Bouton ME (2002). Context, ambiguity, and unlearning: sources of relapse after behavioral extinction. Biol Psychiatry 52: 976-986.

Butler AC, Chapman JE, Forman EM, Beck AT (2006). The empirical status of cognitive-behavioral therapy: A review of meta-analyses. Clin Psychol Rev 26: 17-31.

Byrne SP, Rapee RM, Richardson R, Malhi GS, Jones M, Hudson JL (2015). D-cycloserine enhances generalization of fear extinction in children. Depress Anxiety 32: 408-414.

Craske MG, Kircanski K, Zelikowsky M, Mystkowski J, Chowdhury $\mathrm{N}$, Baker A (2008). Optimizing inhibitory learning during exposure therapy. Behav Res Ther 46: 5-27. 
Craske MG, Treanor M, Conway CC, Zbozinek T, Vervliet B (2014). Maximizing exposure therapy: an inhibitory learning approach. Behav Res Ther 58: 10-23.

Davey GCL (1991). Characteristics of individuals with fear of spiders. Anxiety Res 4: 299-314.

de Jong PJ, Muris P (2002). Spider phobia: interaction of disgust and perceived likelihood of involuntary physical contact. J Anxiety Disord 16: 51-65.

Dunsmoor JE, Murphy GL (2014). Stimulus typicality determines how broadly fear is generalized. Psychol Sci 25: 1816-1821.

Dunsmoor JE, Murphy GL (2015). Categories, concepts, and conditioning: how humans generalize fear. Trends Cogn Sci 19: 73-77.

Dunsmoor JE, Paz R (2015). Fear generalization and anxiety: behavioral and neural mechanisms. Biol Psychiatry 78: 336-343.

Dymond S, Dunsmoor JE, Vervliet B, Roche B, Hermans D (2015). Fear generalization in humans: systematic review and implications for anxiety disorder research. Behav Ther 46: 561-582.

Hamm A (2006). Spezifische Phobien. Hogrefe Göttingen.

Hofmann SG, Smits JAJ (2008). Cognitive-behavioral therapy for adult anxiety disorders: a meta-analysis of randomized placebocontrolled trials. J Clin Psychiatry 69: 621-632.

Hofmann W, De Houwer J, Perugini M, Baeyens F, Crombez G (2010). Evaluative conditioning in humans: a meta-analysis. Psychol Bull 136: 390-421.

Lovibond SH, Lovibond PF (1995). Manual for the Depression Anxiety Stress Scales 2nd edn. Psychology Foundation: Sydney, Australia.

Ludvik D, Boschen MJ, Neumann DL (2015). Effective behavioural strategies for reducing disgust in contamination-related OCD: A review. Clin Psychol Rev 42: 116-129.

Margraf J (1994). Mini-DIPS. Diagnostisches Kurzinterview bei psychischen Störungen. Springer: Heidelberg.

Matchett G, Davey GC (1991). A test of a disease-avoidance model of animal phobias. Behav Res Ther 29: 91-94.

Mineka S, Mystkowski JL, Hladek D, Rodriguez BI (1999). The effects of changing contexts on return of fear following exposure therapy for spider fear. J Consult Clin Psychol 67: 599-604.

Mystkowski JL, Craske MG, Echiverri AM (2002). Treatment context and return of fear in spider phobia. Behav Ther 33: 399-416.

Norton PJ, Price EC (2007). A meta-analytic review of adult cognitive-behavioral treatment outcome across the anxiety disorders. J Nervous Ment Dis 195: 521-531.

Olatunji BO, McKay D (2007a). Disgust and psychiatric illness: have we remembered? Br J Psychiatry 190: 457-459.

Olatunji BO, Smits JAJ, Connolly K, Willems J, Lohr JM (2007b). Examination of the decline in fear and disgust during exposure to threat-relevant stimuli in blood-injection-injury phobia. J Anxiety Disord 21: 445-455.

Öst LG (1997)Rapid treatment of specific phobias. In: Davey GCL (ed). Phobias: A handbook of Theory, Research and Treatment. Wiley: New York. pp 227-246.
Pace-Schott EF, Verga PW, Bennett TS, Spencer RMC (2012). Sleep promotes consolidation and generalization of extinction learning in simulated exposure therapy for spider fear. J Psychiatr Res 46: 1036-1044.

Pössel P, Hautzinger M (2003). Dysfunktionale Überzeugungen bei Spinnenangst. Zeitschrift Klin Psychol Psychother 32: 24-30.

Rinck M, Bundschuh S, Engler S, Muller A, Wissmann J, Ellwart T et al (2002). Reliability and validity of German versions of three instruments measuring fear of spiders. Diagnostica 48: 141-149.

Rodriguez BI, Craske MG, Mineka S, Hladek D (1999). Contextspecificity of relapse: effects of therapist and environmental context on return of fear. Behav Res Ther 37: 845-862.

Rowe MK, Craske MG (1998a). Effects of an expanding-spaced vs massed exposure schedule on fear reduction and return of fear. Behav Res Ther 36: 701-717.

Rowe MK, Craske MG (1998b). Effects of varied-stimulus exposure training on fear reduction and return of fear. Behav Res Ther 36: 719-734.

Ruhmland M, Margraf J (2001a). Effektivität psychologischer Therapien von generalisierter Angststörung und sozialer Phobie: Meta-Analysen auf Störungsebene. Verhaltenstherapie 11: 27-40.

Ruhmland M, Margraf J (2001b). Effektivität psychologischer Therapien von Panik und Agoraphobie: Meta-Analysen auf Störungsebene. Verhaltenstherapie 11: 41-53.

Ruhmland M, Margraf J (2001c). Effektivität psychologischer Therapien von spezifischer Phobie und Zwangsstörung: MetaAnalysen auf Störungsebene. Verhaltenstherapie 11: 14-26.

Schienle A, Walter B, Stark R, Vaitl D (2002). Ein Fragebogen zur Erfassung der Ekelempfindlichkeit (FEE). Z Klin Psychol Psychother 31: 110-120.

Smits JAJ, Telch MJ, Randall PK (2002). An examination of the decline in fear and disgust during exposure-based treatment. Behav Res Ther 40: 1243-1253.

Vervliet B, Craske MG, Hermans D (2013). Fear extinction and relapse: state of the art. Annu Rev Clin Psychol 9: 215-248.

Vervliet B, Vansteenwegen D, Baeyens F, Hermans D, Eelen P (2005). Return of fear in a human differential conditioning paradigm caused by a stimulus change after extinction. Behav Res Ther 43: 357-371.

Vervliet B, Vansteenwegen D, Eelen P (2004). Generalization of extinguished skin conductance responding in human fear conditioning. Learn Mem 11: 555-558.

Vervoort E, Vervliet B, Bennett M, Baeyens F (2014). Generalization of human fear acquisition and extinction within a novel arbitrary stimulus category. PLoS ONE 9: e96569.

Wolpe J (1973). The Practice of Behavior Therapy. Pergamon: New York.

Woody SR, Teachman BA (2000). Intersection of disgust and fear: normative and pathological views. Clin Psychol Sci Pract 7: 291-311.

Woody SR, Tolin DF (2002). The relationship between disgust sensitivity and avoidant behavior: Studies of clinical and nonclinical samples. J Anxiety Disord 16: 543-559. 